\title{
Integrity of the Post-LRR Domain Is Required for TIR-NB-LRR Function
}

\author{
Simon B. Saucet, ${ }^{+}$Daniel Esmenjaud, and Cyril Van Ghelder \\ Institut Sophia Agrobiotech, INRAE, Université Nice Côte d'Azur, Sophia Antipolis 06903, France \\ Accepted 15 November 2020.
}

\begin{abstract}
Plants trigger appropriate defense responses, notably, through intracellular nucleotide-binding (NB) and leucine-rich repeat (LRR)-containing receptors (NLRs) that detect secreted pathogen effector proteins. In NLR resistance genes, the toll/ interleukin-1 receptor (TIR)-NB-LRR proteins (TNLs) are an important subfamily, out of which approximately half the members carry a post-LRR (PL) domain of unknown role. We first investigated the requirement of the PL domain for TNLmediated immune response by mutating the most conserved amino acids across PL domains of Arabidopsis thaliana TNLs. We identified several amino acids in the PL domain of RPS4, required for its ability to trigger a hypersensitive response to AvrRps4 in a Nicotiana tabacum transient assay. Mutating the corresponding amino acids within the PL domain of the tobacco TNL gene $N$ also affected its function. Consequently, our results indicate that the integrity of the PL domain at conserved positions is crucial for at least two unrelated TNLs. We then tested the PL domain specificity for function by swapping PL domains between the paralogs RPS4 and RPS4B. Our results suggest that the PL domain is involved in their TNL pair specificity, 'off state' stability, and NLR complex activation. Considering genetically paired Arabidopsis TNLs, we finally compared the PL and TIR domains of their sensor and executor sequences, respectively. While TIR and PL domains from executors present complete motifs, sensors showed a lack of conservation with degenerated motifs. We here provide a contribution to the functional analysis of the PL domain in order to decipher its role for TNL function.
\end{abstract}

Keywords: effector-triggered immunity, hypersensitive response, $N$ gene, NLR, plant resistance, post-LRR (PL) domain, RPS4, TNL

Plants have the ability to recognize the presence of microbial organisms. This recognition is mediated by components of the plant innate immune system, which is essential to trigger an

${ }^{\dagger}$ Corresponding author: S. B. Saucet; simon.saucet@inrae.fr

Funding: S. B. Saucet has received the support of the European Union in the framework of the Marie-Curie FP7 COFUND People Programme through the award of an AgreenSkills+ fellowship (under grant agreement no. 609398) and from the European Union's Horizon 2020 Marie Skłodowska-Curie Actions research and innovation programme under grant agreement no. 838478 .

*The $\boldsymbol{e}$-Xtra logo stands for "electronic extra" and indicates there are supplementary materials published online.

The author(s) declare no conflict of interest.

(c) (1) () $\odot$ Copyright $\odot 2021$ The Author(s). This is an open access article (C) (1) () () distributed under the CC BY-NC-ND 4.0 International license. appropriate defense response during pathogen invasion. While a variety of transmembrane receptors sense pathogen-associated molecules in the apoplasm, nucleotide-binding (NB)- and leucinerich repeat (LRR)-containing receptors (NLRs) detect pathogen effector proteins secreted intracellularly. Effector recognition can occur through direct protein-protein association with the NLR or when the NLR perceives the molecular disturbance resulting from the effector activity (Jones and Dangl 2006). The immune response triggered by NLR-mediated effector recognition (also known as effector-triggered immunity [ETI]) restricts pathogen growth and may culminate in a hypersensitive response (HR) (Cui et al. 2015). The HR is a strong immune response characterized by programmed cell death, which is accompanied by release of pathogen-disruptive compounds such as reactive oxygen species (Heath 2000).

In plants, genes involved in ETI are inherited from the parents and participate, therefore, in an innate immunity. Genetic variability of individual plants is a limiting parameter toward the capacity of NLRs to recognize pathogen effector variants. The NLRs are multidomain proteins that activate downstream signaling through structural reconfiguration following effector recognition (Takken et al. 2006; Wang et al. 2019a and b). The number and nature of the domains can vary; however, the core NB-LRR structure defines this receptor family. The NB domain induces the reversible shift from inactive to active NLR through exchange of ADP to ATP binding and participates in NLR multimerization (Takken et al. 2006). The LRR domain can be involved in effector recognition and NLR stabilization in a resting state (Takken and Goverse 2012). The N-terminal domain is associated with the activation of downstream signaling pathway. Three NLR subfamilies have been identified according to their N-terminal domain: the coiled-coil (CC)-NB-LRR (CNLs), the toll/interleukin 1 receptor (TIR)-NB-LRR (TNLs), and the RPW8-like-NB-LRR (RNLs) (Shao et al. 2016). The RNL subfamily gathers 'helper' NLRs that are required for all the executor CNLs and TNLs tested so far (Castel et al. 2019; Qi et al. 2018). Following a parallel process, a complex network composed of sensor and helper CNLs, which belong to different clades, have been identified in the Solanaceae family (Wu et al. 2017).

Some particular CNLs and TNLs have integrated protein domains targeted by effectors and therefore become direct sensors of effector presence and activity. These 'sensor' NLRs rely on one or both genetically or functionally paired executor NLRs for downstream immune signaling (Césari et al. 2013, 2014; Narusaka et al. 2009). One extensively studied example is the case of Arabidopsis TNL genes RRS1 and RPS4. These two TNLs are organized in a head-to-head configuration on the genome and function together for at least the recognition of AvrRps4 and PopP2, two effectors from Pseudomonas syringae and Ralstonia solanacerum, respectively (Deslandes et al. 2003; Gassmann et al. 1999; Narusaka et al. 2009). An 
integrated WRKY domain in RRS1 is targeted, as are many other WRKY-containing proteins, by AvrRps4 and PopP2 (Sarris et al. 2015; Le Roux et al. 2015). The sensing of those effectors by RRS1 triggers sequential reconfigurations in its intramolecular interactions, which, in turn, activates RPS4 for downstream signaling (Ma et al. 2018). In this context, RRS1 is the 'sensor' and RPS4 the 'executor.' Besides RRS1/RPS4, the Arabidopsis NLRome also contains eight other pairs of TNLs arranged in a similar configuration (Narusaka et al. 2009), including $R R S 1 B / R P S 4 B$, a paralogous pair of RRS1/RPS4 (Saucet et al. 2015).

A recent breakthrough describes the whole three-dimensional structure of the CNL ZAR1 prior to and after recognition of the effector AvrC. This provides precious data about the interactions occurring between the NLR domains in the 'OFF' and 'ON' states of the receptor (Wang et al. 2019b). While the OFF state presents ZAR1 as a folded monomer, the ON state depicts an unfolded ZAR1 molecule that associates in a pentameric structure called a resistosome (Wang et al. 2019a). Additional studies are necessary to reveal if this mechanism also occurs during activation of other CNLs and NLRs from different subfamilies.

The post-LRR (PL) domain, initially named C-terminal nonLRR domain (CTD), was first described during the identification of the TNL $P 2$ gene, which provides flax rust resistance (Dodds et al. 2001). Later, a particular analysis of the PL domain was carried out in the peach genome (Van Ghelder and Esmenjaud 2016), following the discovery of its presence as multiple copies in the $M a$ TNL gene (Claverie et al. 2011). Throughout the diverse domains composing NLRs, the PL domain has been identified in numerous NLRs from the TNLs subfamily. The PL domain is approximately 150 amino acids long and is always located at the C-terminal end of the LRR domain. It is characterized by several conserved amino-acid motifs (Van Ghelder and Esmenjaud 2016) and its presence in approximately half of plant TNLs suggests an important role in the function of receptors.

In this study, we initiated the functional analysis of the PL domain. We focused on two TNLs, RPS4 and N, both of which carry a well-conserved PL domain. Conveniently, a transient HR assay can be used to rapidly assess their functionality. The $N$ gene was originally cloned from tobacco, in which it provides resistance to tobacco mosaic virus (TMV) with the requirement of the RNL NRG1 (Peart et al. 2005; Whitham et al. 1994). It recognizes the complex formation between p50, a peptide of the TMV helicase protein, and NRIP1, a sulfurtransferase from tobacco through association with its TIR domain (Burch-Smith et al. 2007; Caplan et al. 2008). We reannotated the Arabidopsis TNL repertoire and applied mutations on characteristic signatures of RPS4 and N PL domains. We showed that several mutations affect both RPS4- and N-triggered HR in an effector-independent manner, at least for RPS4, suggesting a common role of the PL domain in TNL function. By swapping PL domains from $R P S 4$ and its paralog RPS4B, we obtained results suggesting that the PL domain is involved in receptor OFF state stability. Finally, we point out the variation and conservation that exists between TIR and PL domain sequences in Arabidopsis sensor and executor genes. Overall, this manuscript depicts a functional analysis dedicated to an underestimated domain, crucial for plant resistance. Our results pave the way for future studies aiming to discover the precise role of the PL domain in TNLmediated immunity in plants.

\section{RESULTS}

\section{The PL domain is widely found in the TNL repertoire from the Arabidopsis thaliana genome.}

We analyzed the distribution and the amino-acid composition of the PL domain in the TNL repertoire of the Arabidopsis genome. By analyzing the region localized downstream the LRR domain with the PL motifs defined by Van Ghelder and Esmenjaud (2016), we found 68 TNL-coding genes containing PL domain signatures. Among the 132 TNL-related sequences (TIR-only included), PL motifs were mainly found in TNLs that display the full canonical TIR-NB-LRR organization (Supplementary Table S1). A motif analysis carried out with these 68 sequences revealed four robust signatures spread out on the domain (Fig. 1). The first motif starts immediately after the LRR domain and contains conserved proline $(\mathrm{P})$ and phenylalanine $(\mathrm{F})$ residues embedded in a characteristic $\mathrm{P}-\mathrm{X}-$ [YEW]-F signature. The second motif, predicted to form a $\beta$-strand, displays a conserved cysteine (C) followed by hydrophobic residues. As for motif 2, motifs 3 and 4 are included in predicted $\beta$-strands and harbor conserved histidine $(\mathrm{H})$ and cysteine-glycine (CG), respectively. The secondary structure analysis revealed a conserved structure that is exclusively made of $\beta$-strands (six in total) mainly localized inside and between motifs. The alignment also revealed a conserved phenylalanine within the fifth $\beta$-strand (Fig. 1). The core PL domain (i.e., containing motifs 1 to 4 ) is made of 130 aa, on average (standard deviation $=9.45 \mathrm{aa}$ ). However, the majority of PLcontaining sequences also harbor size-variable and polymorphic extra C-terminal peptide chains $(>30$ aa), which we did not consider as a part of the core PL domain, and that are predicted to code for either nuclear localization signal (NLS), a transmembrane region, or unknown features. The screening of the full protein dataset in Arabidopsis (Araport11) using our motifs identified four additional PL-related sequences. We found a LRR-PL protein (AT4G19470), two PL-only proteins (AT4G16857, AT4G16957), and one PL-unknown domain (AT5G46500). All these genes are probably the result of incomplete gene duplications. Our motif investigation also highlighted a single TNL that underwent a PL duplication (AT5G46510). In parallel, we produced a hidden Markov model (HMM) profile (discussed below) and used it to search the Araport11 dataset for PL-containing proteins. Our results confirm the findings obtained with the motif analysis, thus providing a list (Supplementary Table S1) of PL-containing TNLs and others lacking PL domains due to either the absence of sequences downstream of the LRR, the presence of other domains, or low similarity with PL motifs or profiles (poor e-values).

The structural annotation of TNLs showed that the majority (74\%) of PL-containing TNLs (TNLPs) do not contain introns between the end of the LRR-encoding exon and the start of the PL-coding exon (Fig. 1, main structural model; Supplementary Table S2). This feature has probably helped to avoid loss of exon coding for PL domains during duplication events, which is a frequent process occurring in TNLs. Interestingly, a small subset of TNLs harbors independent PL-coding exons, among which we found all the executor TNLs or TNL-Bs, as typified by Meyers and colleagues (2003). The proportion of TNLs carrying a PL domain (over 50\%) implies a specific mode of action involving the PL domain shared by many TNLs. Furthermore, and no matter the type of TNL, the presence of strongly conserved amino acids in PL motifs suggests that they might have an important role for TNL function that we will further investigate.

\section{Deletion and mutations within the PL domain strongly impact TNLs activity.}

To analyze the function of the PL domain, we focused on two PL-containing TNLs, RPS4 and N. By comparing these two genes from distant dicotyledonous species, we expected to shed light on common and specific molecular mechanisms in plant TNLs involving the PL domain. Both RPS4 and N belong to the 
NLR executor class. They both carry a C-terminal extension (the CTD), after the PL domain, of about 100 and 30 amino acids for RPS4 and N, respectively. The CTD of RPS4 contains a NLS required for function (Wirthmueller et al. 2007). RPS4 is paired in the genome with RRS1 for function, whereas $\mathrm{N}$ has not been described so far to be physically or functionally paired with another TNL. RPS4- and N-triggered immunity can be reconstituted in an Agrobacterium tumefaciens-mediated transient expression assay in Nicotiana tabacum (Narusaka et al. 2013; Ueda et al. 2006; Williams et al. 2014). In this publication, we have used this assay to assess the functionality of the different RPS4 and $N$ alleles we generated.

We first examined if the PL domain is dispensable for TNL function by constructing a version of RPS4 depleted of its PL domain. Using the Golden Gate kit from The Sainsbury Laboratory (Norwich, U.K.) (Engler et al. 2014), we cloned an RPS4 allele missing the PL domain sequence from F960 to S1118. The wild type (wt) RPS4 allele containing all domains is referred to here as RPS4 ${ }^{\mathrm{TNLPC}}$ (T for TIR, N for NB, L for LRR, $\mathrm{P}$ for PL, and $\mathrm{C}$ for CTD) and the PL-depleted allele as $R P S 4^{\mathrm{TNLC}}$ (Supplementary Fig. S1A). In the transient assay, overexpression of RPS4 ${ }^{\text {TNLPC }}$ together with RRS1 and AvrRps 4 triggered a strong HR (Supplementary Fig. S1B). However, no HR was observed with RPS4 ${ }^{\text {TNLC }}$, suggesting that the PL domain is essential for RPS4 function. Neither overexpression of $R P S 4^{\mathrm{P}}$ alone or in trans with RPS4 ${ }^{\mathrm{TNLC}}$ could reconstitute the HR triggered by wt RPS4 together with RRS1 and AvrRps4. Western blot analysis showed proper accumulation of RPS4 ${ }^{\mathrm{TNLPC}}$, RPS4 ${ }^{\mathrm{TNLC}}$, RPS4 ${ }^{\mathrm{P}}$, and other proteins used in this assay (Supplementary Fig. S2A and C). These results indicate that the presence of the PL domain is essential for RPS4 to function and activate immune signaling.

In regards to the different intensities of macroscopic HR cell death observed in our tobacco transient assays, we propose four levels to rate the HR, i.e., HR, HR-, hr, and no HR (Supplementary Fig. S3). Complete cell death of the infiltrated tissue visible from the upper (Supplementary Fig. S3A) and lower (Supplementary Fig. S3B) surface of the leaf are indicated by HR on the figures as observed with RPS4+RRS1+AvrRps4 (Supplementary Fig. S1B). If the infiltrated area shows patches of tissue cell death visible from the upper and lower faces of the leaf, it is rated HR-. In some cases, only cell death of the lower leaf epidermal surface is observable, which is indicated by hr. Absence of any indication on the leaf picture means absence of macroscopic cell death (no HR).

Following the deletion of the entire PL domain, we analyzed the effect of amino-acid mutations within the PL domain, to identify important residues for function. We chose to mutate, in RPS4, several of the most conserved amino acids identified in PL domain motifs of Arabidopsis (Fig. 1). We applied mutations onto RPS4 PL domain motif 1 (PSWF970-973), motif 2 (G997 and C1001), motif 3 (H1055), conserved phenylalanine F1087, and motif 4 (CG1104-1105) (Fig. 1; Supplementary Fig. S4). We then tested the effect of each mutation on RPS4 activity, using transient HR assay. We expressed RPS4-mutated versions with the $\beta$-glucuronidase gene $(G U S)$ only to test their autoactivity and with $R R S 1+A v r R p s 4$ to test their functionality. In our conditions, RPS4 wt triggered a low intensity of HR (hr) when overexpressed on its own in tobacco (Fig. 2A). The intensity of RPS4-triggered hr we observed in tobacco was weaker than in publications of other authors, who observed a full HR (Huh et al. 2017; Sohn et al. 2014; Zhang et al. 2004). This variation could eventually be explained by the use of different tobacco varieties and growth chamber conditions. However, similar to Sohn et al. (2014) and Huh et al. (2017), RPS4-triggered hr was repressed in the presence of RRS1 but RPS4+RRS1 triggered full HR to AvrRps4 (Fig. 2A). The P-X[YEW]-F signature in motif 1 of the PL domain is widely found in plant TNLPs. Mutation of this signature in RPS4 (RPS4 ${ }^{\text {PSWF970-973AAAA }}$ ) resulted in the complete loss of RPS4 autoactive hr and HR to AvrRps4 (Fig. 2A). We identified that the mutation of the proline (RPS4 ${ }^{\mathrm{P} 970 \mathrm{~A}}$ ) by itself did not abolish the autoactive hr but only produced a hr to AvrRps4. Subsequently, the mutation of the highly conserved phenylalanine (RPS4 $4^{\mathrm{F} 973 \mathrm{~A}}$ ), which marks out the signature, led to a complete loss of autoactive hr and HR to AvrRps4. Previously, the RPS4 ${ }^{\mathrm{G} 997 \mathrm{E}}$ allele was identified in the sushi (suppressor of $\mathrm{slh} 1$ immunity) mutant screen for suppression of the autoimmune slh1 (sensitive to low humidity 1) phenotype (Sohn et al. 2014). This RPS4 ${ }^{\text {G997E }}$ was shown by Sohn and colleagues (2014) to be nonfunctional in the A. tumefaciens HR transient assay, and we reproduced this result with a $R P S 4^{\mathrm{G} 997 \mathrm{E}}$ allele generated in

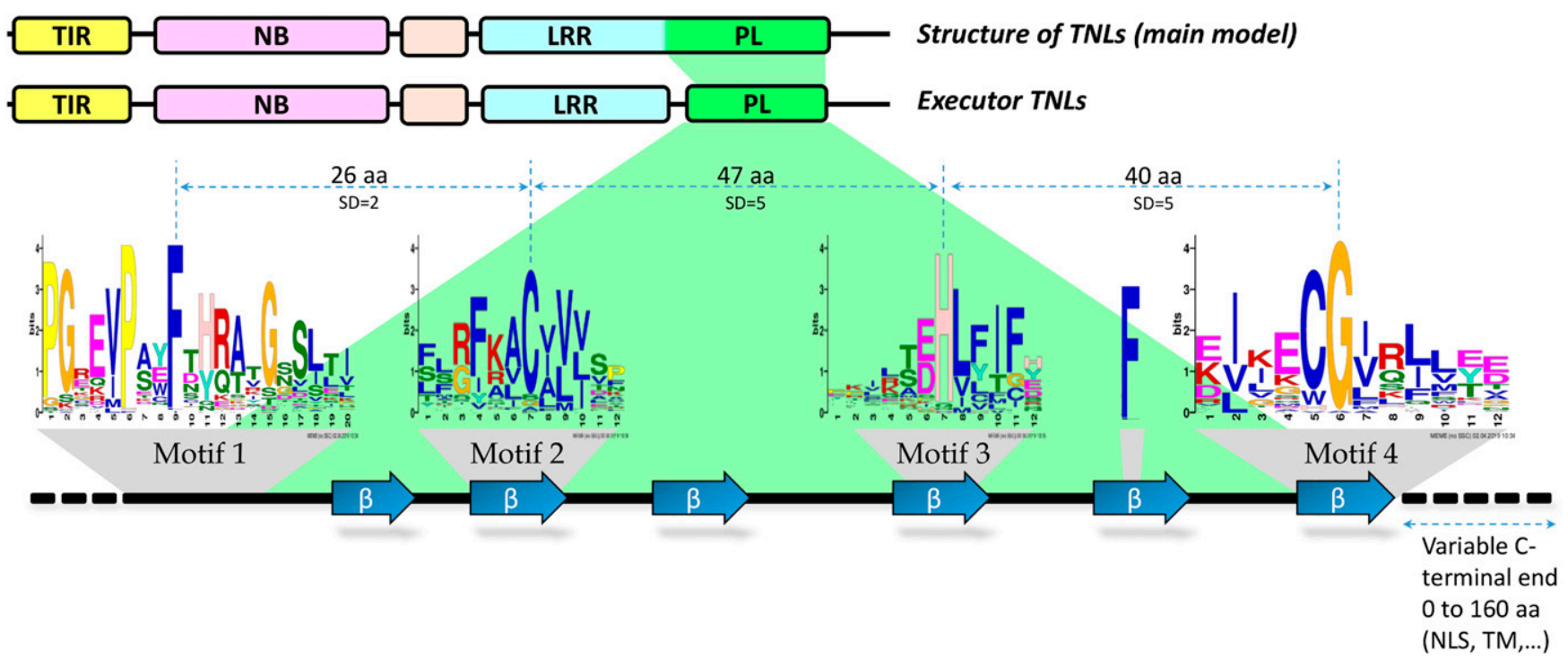

Fig. 1. Structural representation of the post-leucine-rich repeat (PL) domain in TNLs (toll/interleukin-1 [TIR]-nucleotide-binding [NB]-leucine-rich repeat [LRR]) found in the genome of Arabidopsis thaliana. The illustration indicates the motif composition, the average length and standard deviation in amino acids between key conserved residues, and the predicted localization of six $\beta$-strands (blue arrows). A conserved phenylalanine residue (F) is embedded in the fifth $\beta$-strand. NLS $=$ nuclear localization signal and TM $=$ transmembrane. 
our laboratory (Fig. 2A). Interestingly, among Arabidopsis TNLPs, approximately a third show a glycine at this specific amino-acid position and half present an arginine (R). When RPS4 G997 was mutated to an arginine, a glutamic acid, or an alanine (RPS4 ${ }^{\mathrm{G} 997 \mathrm{R}}$, RPS4 ${ }^{\mathrm{G} 997 \mathrm{E}}$, RPS4 ${ }^{\mathrm{G} 997 \mathrm{~A}}$ ), the corresponding RPS4 alleles were nonfunctional, suggesting the requirement of a glycine specifically at this position. In addition, we showed that all the glycine 997 mutated alleles lost the mild autoactivity of RPS4 wt (Fig. 2A). Although we applied mutations on RPS4 corresponding to the most conserved amino acids in Arabidopsis PL domains, some did not alter (RPS4 ${ }^{\mathrm{C} 1001 \mathrm{~A}}$, $\mathrm{RPS}^{\mathrm{C} 1104 \mathrm{~A}}$, RPS4 ${ }^{\mathrm{G} 1105 \mathrm{~A}}, \mathrm{RPS} 4^{\mathrm{H} 1055 \mathrm{~A}}$ ) or altered partially its

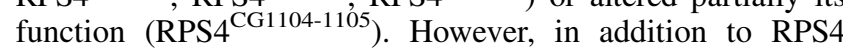
F973, we identified another phenylalanine (RPS4 F1087) that, when mutated (RPS4 ${ }^{\mathrm{F} 1087 \mathrm{~A}}$ ), was strictly deleterious for RPS4 autoactivity and HR to AvrRps4. Throughout the RPS4 mutation tested, we only noticed a difference in the HR intensity levels but no delay in the apparition of these phenotypes.

We then performed conductivity measurements in leaves expressing the different RPS4 mutants. This enabled us to quantify ion leakage during leaf tissue cell death associated with HR to AvrRps4 (Supplementary Fig. S5). Our results correlated with the macroscopic HR we observed. A similar level of conductivity was observed between RPS4 wt and RPS4 mutated alleles showing HR to AvrRps4 (RPS4 ${ }^{\mathrm{C} 1001 \mathrm{~A}}$, $\left.R P S 4^{\mathrm{C} 1104 \mathrm{~A}}, R P S 4^{\mathrm{G} 1105 \mathrm{~A}}, \operatorname{RPS} 4^{\mathrm{H} 1055 \mathrm{~A}}\right)$. The RPS4 ${ }^{\mathrm{P} 970 \mathrm{~A}}$ allele showing hr to AvrRps4 also presented an intermediary level of conductivity. Accordingly, the RPS4 alleles showing no macroscopic HR presented no increase in conductivity over time.

In order to clarify whether each mutated amino acid participates in immune signaling independently of AvrRps4 recognition, we overexpressed RPS4 mutants together with the constitutively active $R R S 1^{\mathrm{SLH} 1}$ allele (Fig. 2A; Supplementary Fig. S2B). A strong cell death was observed in the leaves coexpressing RPS4 and RRS1 $1^{\mathrm{SLH} 1}$, but this phenotype was completely abolished by the RPS4 ${ }^{\mathrm{G} 997 \mathrm{E}}$ mutation (Sohn et al. 2014). In the same manner, RPS4 ${ }^{\text {G997R }}$ and RPS4 ${ }^{\text {G997A }}$ showed no HR in the presence of RRS1 ${ }^{\mathrm{SLH} 1}$. Similar to the immune response to AvrRps4 together with RRS1 ${ }^{\text {SLH1 }}$, RPS4 PSWF970-973AAAA, RPS4 ${ }^{\text {F973A }}$, and RPS4 ${ }^{\text {F1087A }}$ showed no HR, RPS4 ${ }^{\text {P970A }}$ showed hr, RPS4 ${ }^{\text {C1001A }}$, RPS4 $4^{\mathrm{C} 1104 \mathrm{~A}}$, RPS4 ${ }^{\mathrm{G} 1105 \mathrm{~A}}$, and RPS4 $4^{\mathrm{H} 1055 \mathrm{~A}}$ triggered HR, and RPS4 ${ }^{\mathrm{CG} 1104-1105}$ was HR-. Altogether, this result suggests that the RPS4 mutants tested are, rather, altered in signaling events downstream of AvrRps4 recognition.

To test the importance of conserved amino acids in PL domains across plant species, we investigated the impact of several of the mutations tested on RPS4 onto the TNL N. Coexpression of $N$ with $P 50$ in $N$. tabacum triggered a strong HR (Fig. 2B). We used N. tabacum cv. Xanthi in our assay, as it does not show HR to P50. No HR was observed with the coexpression of $N$ and $G U S$. Following sequence comparison (Supplementary Fig. S4), we generated $N$ alleles presenting, separately, the mutations $\mathrm{N}^{\mathrm{F} 984 \mathrm{~A}}, \mathrm{~N}^{\mathrm{G} 1009 \mathrm{E}}$, and $\mathrm{N}^{\mathrm{F} 1088 \mathrm{~A}}$, corresponding to RPS4 $4^{\mathrm{F} 973 \mathrm{~A}}$, RPS4 $4^{\mathrm{G} 997 \mathrm{E}}$, and RPS4 $4^{\mathrm{F} 1087 \AA}$, respectively. Those three mutations abolished $N$-mediated HR in $N$. tabacum as they did for RPS4 (Fig. 2B). We confirmed the production and accumulation of each mutated RPS4 and N protein using Western blot (Supplementary Fig. S2D, E, and F). We also generated $N$ alleles with the mutations $\mathrm{N}^{\mathrm{P} 981 \mathrm{~A}}$, $\mathrm{N}^{\mathrm{C} 1013 \mathrm{~A}}$ and $\mathrm{N}^{\mathrm{G} 1096 \mathrm{~A}}$, corresponding to RPS4 ${ }^{\mathrm{P} 970 \mathrm{~A}}$,

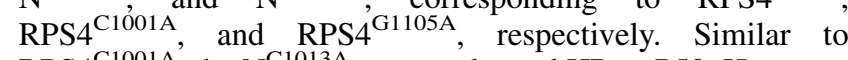
RPS4 ${ }^{\mathrm{C} 1001 \mathrm{~A}}$, the $\mathrm{N}^{\mathrm{C} 1013 \mathrm{~A}}$ mutant showed HR to P50. However, $\mathrm{N}^{\mathrm{P} 981 \mathrm{~A}}$ triggered a full HR to P50 when RPS4 ${ }^{\mathrm{P} 970 \mathrm{~A}}$ responded with a hr to AvrRps4. Conversely, $\mathrm{N}^{\mathrm{G} 1096 \mathrm{~A}}$ showed HR- to P50 when the corresponding mutation, by itself, in RPS4 ${ }^{\mathrm{G} 1105 \mathrm{~A}}$ had no impact. Overall, our data revealed conserved amino-acid positions in two TNL PL domains that are fully and partially required for receptor function.

\section{The PL domain is involved in TNL OFF-state stability.}

In Arabidopsis, the TNL RRS1B/RPS4B pair has been identified as a paralog of RRS1/RPS4 (Saucet et al. 2015). Although both pairs recognize AvrRps4, RRS1B/RPS4B does not recognize PopP2. The two pairs show high architecture and aminoacid sequence similarities (RRS1/RRS1B, 58\% and RPS4/ RPS4B, 64\%), but each TNL only functions with its respective pair partner. It was shown that swapping RPS4 and RPS4B TIR domains is not a limiting factor for function, suggesting that the pair specificity relies on other domains (Saucet et al. 2015). We performed a similar analysis to test the importance of the PL domain for functional pairing. We therefore constructed chimeras between RPS4 and RPS4B by swapping their PL domains only or in combination with their CTD and TIR domains (Fig. 3A; Supplementary Fig. S4). We next refer

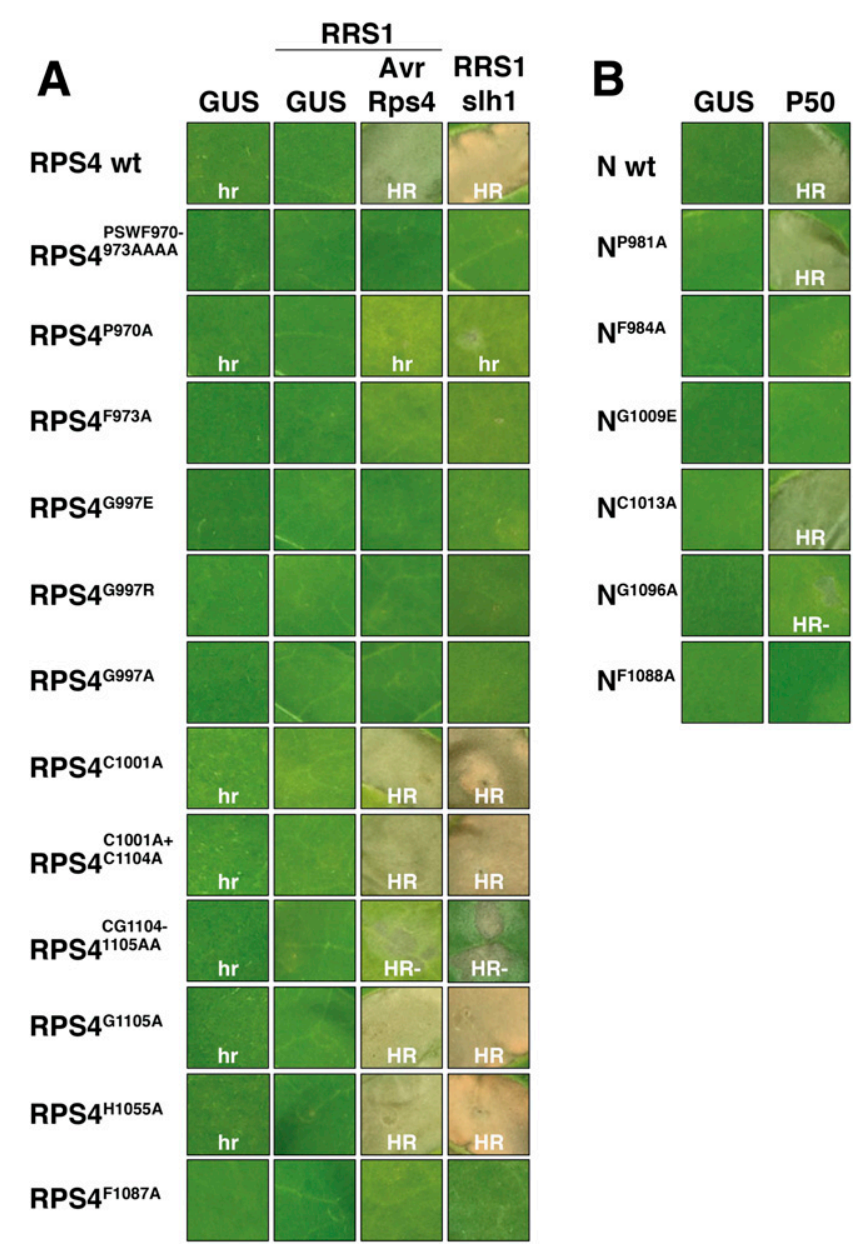

Fig. 2. Different mutations within post-leucine-rich repeat (PL) domains affect the ability of RPS4 and N to trigger a hypersensitive response (HR) in Nicotiana tabacum. HR assay in N. tabacum leaves using wild type or mutated alleles of RPS4 or N. A, N. tabacum leaves were coinfiltrated with different combinations of Agrobacterium tumefaciens for overexpression of the $\beta$-glucuronidase gene $(G U S), R R S 1$ with either AvrRps 4 or GUS, and $R R S 1^{\mathrm{SLH} 1}$ together with different $R P S 4$ variants, and $\mathbf{B}, P 50$ or GUS together with different $N$ variants. HR refers to complete death of the infiltrated leaf tissue, whereas HR- refers to patches of tissue death and hr to death of lower epidermal cell layer. Equivalent amino-acid positions in PL motifs between RPS4 and N are P970-P981, F973-F984, G997-G1009, C1001-C1013, G1105-G1096, F1087-F1088. Pictures of infiltrated leaves were taken at 5 days postinfiltration. 
to each domain (in the following order TIR, NB, LRR, PL, CTD) using the letter A or B, depending whether they belong to RPS4 (pair A) or RPS4B (pair B), respectively. For example, RPS4 wt will be referred as RPS4(AAAAA) and RPS4B RPS4(BBBBB).

We first expressed the different chimeras together with $G U S$ only in order to establish their potential autoactivity. The protein production of each chimera was confirmed in infiltrated leaves by Western blot (Supplementary Fig. S2G). The wt RPS4(AAAAA) triggered hr when overexpressed on its own but no HR was observed for RPS4(BBBBB) (Fig. 3B). Strikingly, RPS4(AAABA) showed a strong autoactive immune phenotype with a rapid HR. In RPS4(AAABB), extending the

A

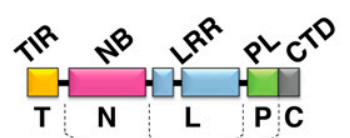

RPS4 (A A A A )

RPS4B (B B B B B)

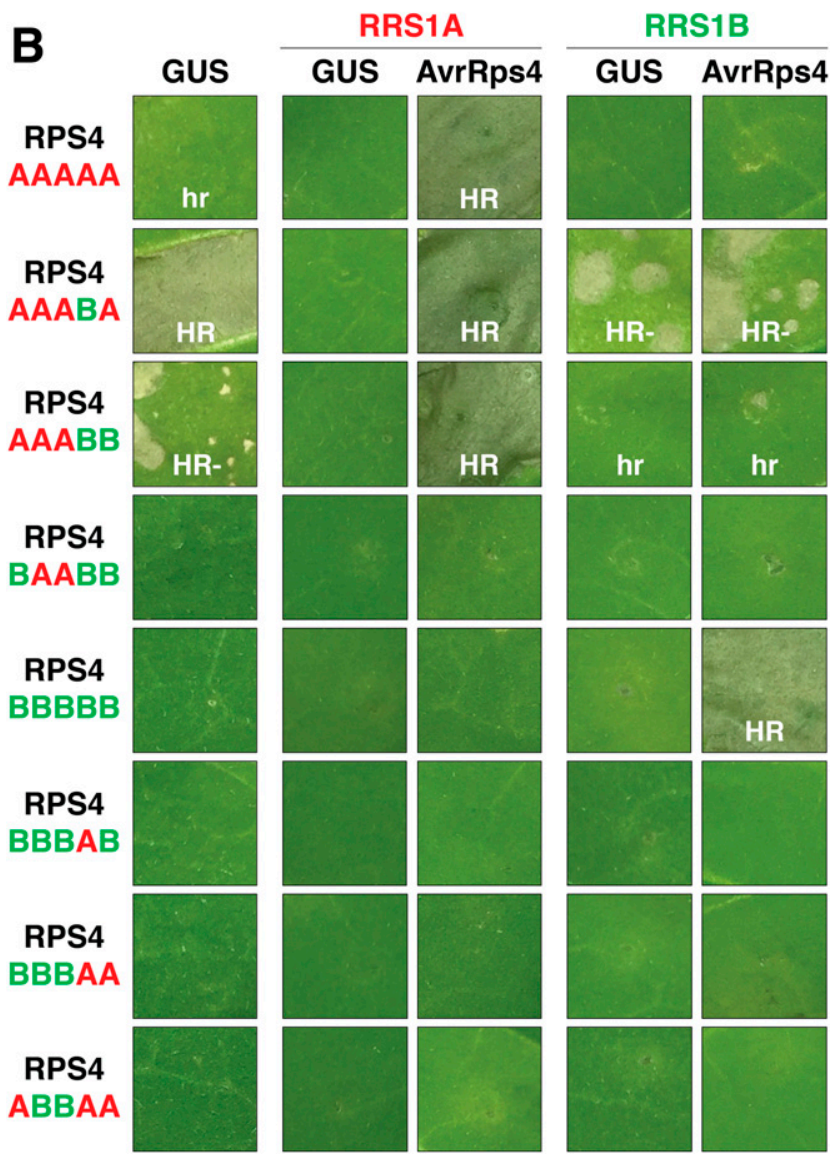

Fig. 3. Post-leucine-rich repeat (PL) domain swap between RPS4 and RPS4B results in autoactive or nonfunctional chimeras. A, Schematical representation of RPS4 and RPS4B gene architecture. Each box represents exonic sequences coding for particular protein domains. $\mathrm{T}=$ toll/ interleukin, $\mathrm{N}=$ nucleotide binding, $\mathrm{L}=\mathrm{LRR}, \mathrm{P}=\mathrm{PL}, \mathrm{C}=\mathrm{C}$ terminal. $\mathbf{B}$, Hypersensitive response (HR) assay in Nicotiana tabacum leaves using Agrobacterium tumefaciens transformation. Each leaf was coinfiltrated to overexpress different combinations of RRS1 or RRS1B with AvrRps 4 or the $\beta$-glucuronidase gene $(G U S)$ together with wild type or different $R P S 4 / R P S 4 B$ chimeric alleles. HR refers to complete death of the infiltrated leaf tissues, whereas HR- and hr refer to lower levels of tissue cell death. Letters in red and green indicate whether the protein domains belong to pair A (RRS1/RPS4) or pair B (RRS1B/RPS4B), respectively. Pictures were taken 5 days postinfiltration.
CTD from RPS4B diminished the intensity of RPS4(AAABA) autoactivity with HR-. Moreover, addition of the TIR domain of RPS4B in RPS4(BAABB) chimera completely abolished the HR. This result suggests that exchanging the PL domain destabilizes RPS4 toward an ON state but the interaction with at least the CTD and, probably, also the TIR domain from the same TNL restores stability toward an OFF state. The fact that overexpression of RPS4 full length triggers hr and RPS4 TIR domain HR whereas RPS $4 B$ full length and TIR domain trigger no HR (Saucet et al. 2015; Swiderski et al. 2009; Zhang et al. 2004) could also explain why RPS4(BAABB) did not show autoactive cell death phenotype. Surprisingly, none of the RPS4(BBBAB), (BBBAA), and (ABBAA) chimeras showed autoactivity when overexpressed with $G U S$. This suggests the NB-LRR domain of RPS4B is able to maintain the receptor in an OFF state, as RPS4(ABBAA) did not show autoactivity despite the presence of RPS4 TIR domain.

Similarly to the results shown in Figure 2A, RPS4(AAAAA)triggered hr was abolished with the coexpression of RRS1 (Fig. $3 \mathrm{~B})$. This phenomenon is the result of a stabilizing/repressing effect that RRS1 applies on RPS4 through direct proteinprotein interaction and possibly TIR-TIR domain interaction (Huh et al. 2017; Williams et al. 2014). As a result, both the autoactive $\mathrm{HR}$ and $\mathrm{HR}$ - triggered by RPS4(AAABA) or (AAABB) were suppressed in the presence of RRS1 with no HR. This suggests that RRS1 is able to repress effectively different levels of RPS4 chimera autoactivation. Interestingly, RRS1B was also able to repress RPS4(AAAAA), (AAABA), and (AAABB) autoactive phenotypes into no HR, HR- and hr, respectively. Our results indicate that RRS1 and, to a lesser extent, RRS1B can act on those chimeras to maintain them toward an OFF state.

When coexpressed with RRS1 and AvrRps4, RPS4(AAABA) and (AAABB) triggered an HR in N. tabacum (Fig. 3B). This suggests that, following AvrRps4 recognition, RRS1 alleviates its repression onto the RPS4(AAABA) chimera. In the case of RPS4(AAABB), the HR observed in the presence of RRS1+AvrRps4 was stronger than when overexpressed on its own (HR-) suggesting that RRS1 is able to activate it, to a certain degree, after AvrRps4 perception. However, we observed no HR with RPS4(BAABB)+RRS1+AvrRps4, suggesting either that the TIR and PL-CTD combination from RPS4B is not inclined to proceed to the activation triggered by RRS1mediated AvrRps4 recognition or simply that RPS4(BAABB) is not functional. Oppositely, the intensity of the HR observed when RPS4(AAABA) and (AAABB) were overexpressed with $R R S 1 B+A v r R p s 4$ did not increase compared with $R R S 1+G U S$. In addition, RPS4(AAAAA) autoactive hr was not restored with RRS1B+AvrRps4. This indicates that the repressive effect of RRS1B on RPS4(AAAAA) as well as on RPS4(AAABA) and (AAABB) chimeras still applied even after AvrRps4 perception. This also shows that RRS1B is unable to activate these chimeras after AvrRps4 recognition; in the same manner, it does not activate RPS4. RPS4(BAABB) together with RRS1B did not show HR to AvrRps4, suggesting that the TIR, PL, and CTD of RPS4B are not sufficient to provide functional pairing with RRS1B or, again, that this chimera is nonfunctional. None of the chimeras presenting the NB-LRR domains from RPS4B [RPS4(BBBAB), RPS4(BBBAA), and RPS4(ABBAA)] were autoactive nor functioning with either RRS1 or RRS1B in the presence of AvrRps4. This result indicates that the NB-LRR domain from RPS4B is not prone to function with at least a foreign PL domain. This might be also true in a context of potential autoactivity, as RPS4(ABBAA) did not show HR despite the presence of the RPS4 TIR domain. In the case of RPS4B, PL domain integrity is therefore essential for function and possibly also for specific pairing with RRS1B. 


\section{Paired TNL sensors present degenerated TIR and} PL domains.

Considering the particular mode of function of paired TNLs, we compared the PL domains of this particular class with the rest of the TNL repertoire. As previously evoked in the phylogenetic analyses based on the NB and TIR domains (Meyers et al. 2002, 2003), the phylogeny produced using the PL domain gathered the executor TNLs in the same clade (clade 4) (Fig. 4A; Supplementary Fig. S6). The tree topology is mainly explained by the polymorphism observed in motifs 1 and 2, and the longer length of the core PL (motifs 1 to 4 ) in the sequences of clade 4 compared with the other TNLPs (on average 20 amino acids longer). In the sequence from clade 4 , we identified, in motif 1 , a conserved tryptophan before the F residue instead of $Y$ or $E$ residues. Similarly, we observed a conserved glycine in motif 2 , which is crucial for RPS4-triggered HR (Fig. 2A), whereas the other sequences mostly displayed an arginine (Fig. 4A). The sensor TNLs (TNL-A type), which underwent domain reorganization, domain fusion, or loss (Fig. 4B), did not seem to contain proper PL domains. Indeed, we only identified remainders of PL motifs in the sequences located immediately after the LRR domain. More specifically, the absence of motif 1 and conserved residues in other motifs, at least, or even the complete absence of PL motifs in some cases indicate a putative degeneration of the PL domain (Fig. 4C). Moreover, the TIR domains of sensor TNLs also present modified TIR motifs, particularly in their $\mathrm{N}$ terminus and in the region localized immediately upstream of the NB P-loop motif (Supplementary Fig. S7). Our analysis points out the co-occurrence of degenerated TIR and PL domains in paired sensor TNLs in Arabidopsis.
In an attempt to test if the degenerated TIR and PL domains are responsible for the RRS1 inability to trigger downstream signaling on its own after perception of AvrRps4, we swapped its TIR and PL domains with the ones from RPS4 (Supplementary Fig. S8A). Despite detecting all the chimeras in a Western blot, none of the single and combined swaps gave the chimeric RRS1-RPS4 alleles the ability to trigger cell death on its own in response to AvrRps4 (Supplementary Figs. S2B and S8B). This result suggests that carrying conserved and complete TIR and PL domains from an executor TNL is not sufficient for a sensor TNL to become a sensor-executor. The $R R S 1^{\mathrm{TNL}}-R P S 4^{\mathrm{P}}-R R S 1^{\mathrm{WC}}$ alleles showed autoactivity when overexpressed with RPS4, similar to what has been shown in another study with the whole PL-encoding exon swap (Ma et al. 2018). Interestingly, the two other chimeras RPS4 ${ }^{\mathrm{T}}-R R S 1^{\mathrm{NLPWC}}$ and $R P S 4^{\mathrm{T}}-R R S 1^{\mathrm{NL}}-R P S 4^{\mathrm{P}}-R R S 1^{\mathrm{WC}}$ were unable to suppress the autoactive HR triggered by RPS4 compared with RRS1 wt. This supports previous findings and suggests that this is mainly RRS1 TIR that acts in this suppression (Williams et al. 2014).

\section{DISCUSSION}

\section{Critical amino-acid positions of PL domains are fully and partially required for TNL function.}

So far, the PL domain was not considered as a domain on its own and it was included within the C-terminal extension of the LRR domain that was simply called CTD. In a recent publication, RPS4 $\triangle$ CTD referred to RPS4 depleted of the whole protein sequence following the LRR, i.e., which lacked 73 amino acids from the LRR-encoding exon as well as the full
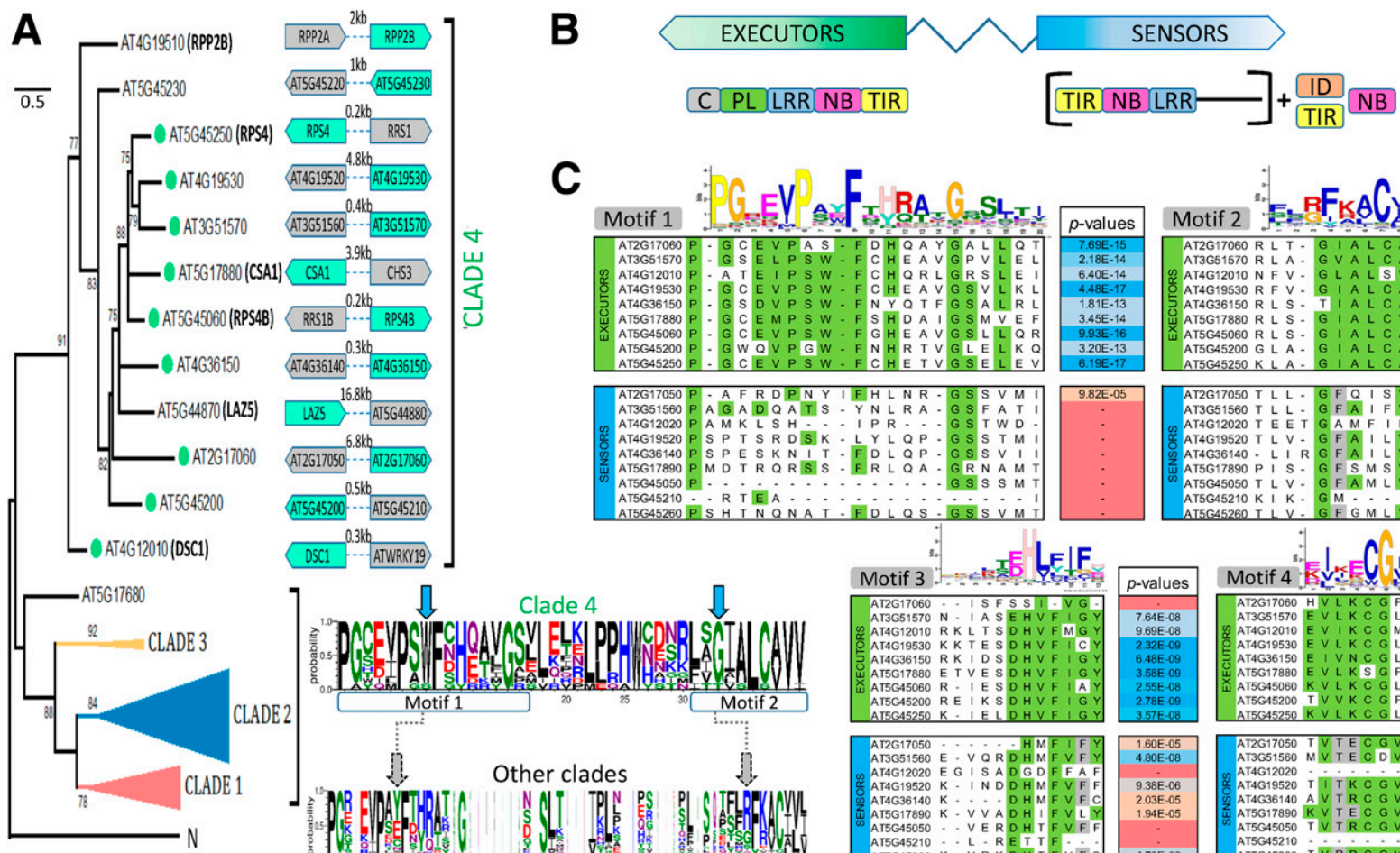

\section{C}
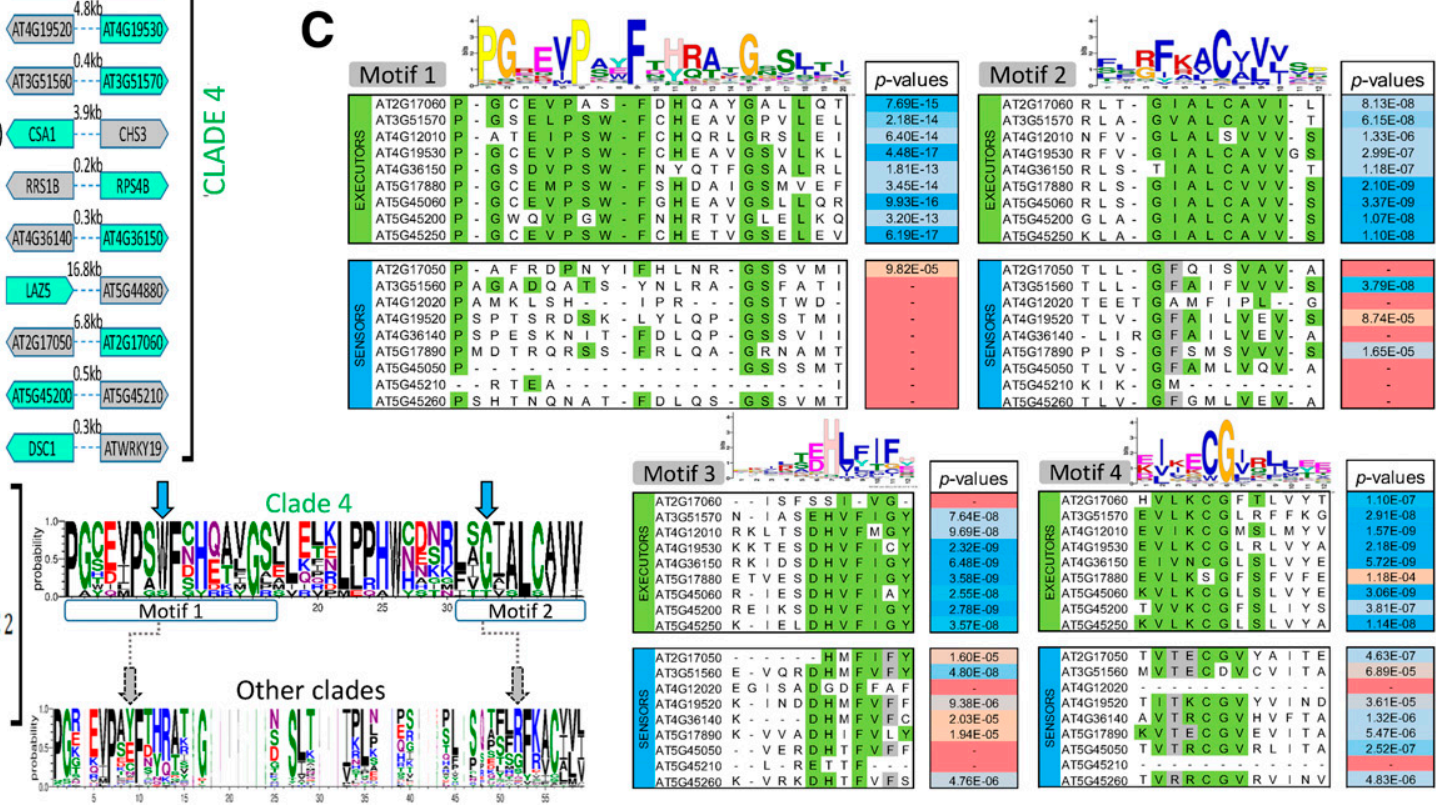

Fig. 4. Comparison of the post-leucine-rich repeat (PL) domain in executor, sensor, and other toll/interleukin 1 (TIR)-nucleotide-binding (NB)-leucine-rich repeat (LRR) receptors (TNLs). A, Maximum likelihood tree based on 68 PL domains (SH-like branch supports $>70$ are shown), using the PL of N as outgroup. Physical distance in the genome between TNL pairs of clade 4, which are either in tandem duplication or in head-to-head position (green dots), are indicated in kilobases. TNLs that display PL domains with clear motifs are indicated in green, others are shown in gray. Consensus sequences corresponding to the motif 1 to 2 region of the sequences from clade 4 and from clades 1,2,3. Blue arrows indicate key residues of clade 4 with their corresponding residues (gray arrows) in clades 1 to 3 consensus sequence. B, Schematic representation of head-to-head pairs of TNLs including executor TNLs with conserved TIR-NB-LRR-PL organization and sensor TNLs displaying integrated domains (ID) and canonical domain (TIR, NB) reorganization. $\mathrm{C}=\mathrm{C}$ terminal. $\mathbf{C}$, Assessment of the four PL motifs in executor and sensor TNLs using the Find Individual Motif Occurrences (FIMO) program $\left(P\right.$ values $<10^{-5} ; P$ value of a motif occurrence is defined as the probability of a random sequence of the same length as the motif matching that position of the sequence with an equivalent or better score). 
amino-acid sequence encoded by the fifth exon containing the PL domain (Ma et al. 2018). This RPS4 $\Delta$ CTD was unable to trigger cell death in tobacco compared with full-length RPS4. Having defined the PL domain and characterized its conservation and importance, we propose to now call CTD the Cterminal extension of the PL domain. In our truncation experiment, we deleted amino acids F960 to S1118 in RPS4, which encompasses the PL domain. We have shown that, despite carrying its CTD, RPS4 ${ }^{\text {TNLC }}$ is not functional without its PL domain. Interestingly RPS4 CTD includes a NLS required for function but this feature is not sufficient to confer functionality to RPS4 ${ }^{\text {TNLC }}$ (Wirthmueller et al. 2007).

Through Arabidopsis NLRome analysis we identified highly conserved amino acids within the different motifs of the PL domain. One common feature of plant PL domains is a P-X[YEW]-F signature in motif 1 . We showed the importance of this row of four amino acids in RPS4 and succeeded in highlighting different phenotypes by mutating P970 and F973. As for RPS $4{ }^{\text {W972 }}$, a tryptophan is usually present in this signature among plant PL domains (Van Ghelder and Esmenjaud 2016). However, in Arabidopsis, this specific position is mainly occupied by either a tyrosine (35\% of PL domains) or a glutamic acid $(22 \%)$ and, to a lesser extent, a tryptophan (19\%), which is the case of executor TNLs. Future experiments performed on these specific residues might provide information about the implication of this polymorphism observed in Arabidopsis compared with other plants. The RPS4 G997 (motif 2) is conserved in $30 \%$ of Arabidopsis PL domains, while $47 \%$ of them present an arginine. RPS4 G997R was equally nonfunctional, suggesting a divergence at this specific residue position between the two main PL-containing TNL groups mentioned above. In addition, we identified RPS4 F973 (motif 1) and F1087 (between motifs 3 and 4), which are required for TNL function. They are conserved at 97 and $87 \%$ among Arabidopsis PL domains, respectively, thus reinforcing the importance of these residues. Considering that we have not noticed an increase of HR with the nonfunctional PL-mutated RPS4 alleles expressed with $R R S 1^{\mathrm{SLH} 1}$ compared with $R R S 1+A v r R p s 4$, we infer that the corresponding amino acids are AvrRps4 recognition-independent. Strikingly, mutating the corresponding three amino acids in $\mathrm{N}$ also abolished its function. Although we noticed subtle differences in the phenotypes of a few mutant alleles in RPS4 and N (RPS4 ${ }^{\mathrm{P} 970 \mathrm{~A}}$ versus $\mathrm{N}^{\mathrm{P} 981 \mathrm{~A}}$ and RPS4 ${ }^{\mathrm{G} 1105 \mathrm{~A}}$ versus $\mathrm{N}^{\mathrm{G} 1096 \mathrm{~A}}$ ), the more drastic phenotypes were confirmed in both gene models, thus reinforcing our findings. Our results suggest a crucial role of these amino-acid positions across plant TNLs, independently of their specific modes of action. Future structural studies will help to localize surface-exposed residues and thus, deepen our understanding of the role of these specific amino acids in the molecular mechanism involving the PL domain.

\section{Unlike point mutations, the PL swap provokes RPS4 autoactivity.}

To test if the PL domain orchestrates the partner specificity for function, we swapped RPS4 and RPS4B PL domains alone or together with the C-terminal extensions and TIR domains. When tested in $N$. tabacum transient assay, RPS4 carrying RPS4B PL domain was autoactive but this autoactivity gradually disappeared after addition of RPS4B CTD and TIR domain. The reduction of HR intensity between RPS4(AAABA) and RPS4(AAABB) suggests that the combination of PL and CTD from the same TNL stabilizes the receptor. In a recent study, a similar construct to RPS4(AAABB) was shown to be functional for the recognition of AvrRps4 and PopP2 in N. tabacum (Ma et al. 2018). Accordingly, RPS4(AAABB) construct showed increased HR compared with its own autoactivity when overexpressed with RRS1 and AvrRps4, suggesting that the PL-CTD from RPS4B can, to some extent, function with RRS1.

In contrast, RPS4(BBBAA) and (BBBAB) were not autoactive nor functional in association with RRS1 or RRS1B. This lack of function cannot be attributed to the required amino acids in the PL domain identified in RPS4 (P970, F973, G997, and F1087), as they are identical in RPS4B. The TIR domain of RPS4B carries a conserved putative catalytic residue (ESTWCLRELAMIKDC) for cleavage of nicotinamide adenine dinucleotide, which excludes this possibility for lack of autoactivity (Wan et al. 2019). Interestingly, RPS4(ABBAA) was not autoactive, suggesting that chimera autoactivity is not only due to the presence of the RPS4 TIR domain but also involves particular intra and also, perhaps, intermolecular interactions.

RPS4(BBBAB) and (BBBAA) did not trigger HR in association with RRS1 or RRS1B in the presence of AvrRps4, suggesting that RPS4 PL or PL-CTD domains are not sufficient to confer compatibility between RPS4B and RRS1 and, oppositely, it could provide an incompatibility for function with RRS1B. A previous study showed that RPS4B carrying the RPS4 TIR domain is functional with RRS1B and similarly with RPS4 and RRS1 (Saucet et al. 2015). This showed that, at least, the TIR and NB-LRR combination from different paralogs was not restrictive for function. However, in the presence of AvrRps4, RPS4(BAABB) and (ABBAA) were not functional with RRS1 nor with RRS1B, suggesting an incompatibility between the NB-LRR and PL-CTD from RPS4 and RPS4B paralogs. The autoactivity of RPS4(AAABA) reinforces the idea that an incompatibility exists between nonhomologous NB-LRR and PL domains, making all chimeras having this combination autoactive or nonfunctional, possibly through one or more negative constraints for protein folding, activation, multimerization, or signaling.

Altogether, our results did not provide a clear answer for the involvement of the PL domain in pair specificity, but they suggest an important role in the stability and function of TNL receptors. Biochemical analysis of domain boundaries within and between pairs will help to resolve their respective molecular compatibility status. In addition, the HR intensity triggered by many NLRs also relies on their compatibility with helper NLRs (Wu et al. 2017). Several TNLs require RNLs, like one or both NRG1 and ADR1, to mediate immune signaling. While both RRS1/RPS4 and RRS1B/RPS4B signal via the RNL NRG1, the status regarding ADR1 is more complex (Castel et al. 2019). Indeed, the autoimmune phenotype conferred by the autoactive allele of RRS1 is partially suppressed in Arabidopsis ADR1 mutants (Dong et al. 2016) and we do not know if RRS1B/RPS4B signals via ADR1. Thus, we cannot infer the real impact of ADR1 and NRG1 in tobacco transient assays regarding the different $\mathrm{HR}$ phenotypes observed with the Arabidopsis pairs RRS1/RPS4 and RRS1B/RPS4B.

Domain swap experiments of membrane-associated pathogen recognition receptors successfully produced functional receptors. For example, swapping the transmembrane and intracellular kinase domain of EFR with the corresponding domains of either FLS2, WAK1, or Xa21 resulted in chimeras still capable of Elf18 recognition and associated PTI (Albert et al. 2010; Brutus et al. 2010; Holton et al. 2015). Similarly, a chimera of FLS2 or WAK1 ectodomain fused to EFR transmembrane and kinase domains was able to trigger PTI in response to flg22 and oligogalacturonides, respectively (Brutus et al. 2010). On the other hand, plant NLRs seem hardly amenable to domain exchange. Similar to our results, domain swap experiment between the CNLs Rx and Gpa2 produced, in majority, autoactive or nonfunctional alleles (Rairdan and 
Moffett 2006). The TNLs L6, L2, and L10 present a variable and short region in the C-terminus, right after the LRR, of about 20 amino acids. Interestingly, these sequences do not present any conserved or degenerated PL motifs. However, swapping L6 C-terminal amino-acid sequence with L2 or L10 generated autoactive or unfunctional chimeras, respectively (Howles et al. 2005), which is very similar to what we observed with RPS4 and RPS4B PL domain swap. Altogether, these results suggest a coevolution between polymorphic domains of NLRs to confer proper intramolecular interactions allowing a safe and reversible activation/inactivation switch but limiting their plasticity for function.

\section{Widely found in Arabidopsis TNLs, the PL domain has been selected in angiosperms.}

TNLs emerged in green algae (Charophytes spp.) (Gao et al. 2018), probably from domain fusion (Yue et al. 2012), and underwent expansions in some bryophytes (Sphagnum fallax) (Xue et al. 2012). On the other hand, the PL domain, which is absent in green algae, bryophytes, and gymnosperms (with the exception of Ginkgo biloba) (Van Ghelder et al. 2019), is found in the large majority of TNLs in dicots and, possibly, in basal angiosperms. Based on TNL functional annotation, 40 to $80 \%$ of eudicot TNLs display a PL domain regardless their respective TNL contingents (Van Ghelder and Esmenjaud 2016). Thus, canonical TNLPs may function in a different manner in modern dicots than TNLs in archaic plants. Except for a few PL-only proteins, which are probably the result of incomplete tandem duplication, the PL domain appears to be exclusively localized in the C-terminal of LRR domains of TNLs. Consistent with this feature, no PL domains have been detected in monocots, as the TNL subfamily has been lost in this phyletic group (Tarr and Alexander 2009). The PL domain polymorphism and the small size of its signatures make more complex the possibility to determine whether the PL domains were de-novo formed or formed by recombination with preexisting archaic PL domains, as a putative motif 1 has been detected embedded in a few LRR-kinase proteins of archaic plant lineage (Supplementary Fig. S9). While the canonical TNL domains (TIR, NB, and LRR) benefited from the knowledge obtained from animal models (e.g., toll receptors or NACHT domains) to decipher their respective functions, the role of the PL domain, which is restricted to plant TNLs, still needs to be revealed. The distribution of the PL domain in TNLs is not in line with the decoy model (Kroj et al. 2016) but, most probably, with a generic function providing an advantage in the immune mechanism initiated by TNLs in dicots. On the contrary, rare TNLs underwent PL domain duplication as the VICTL of unknown function in Arabidopsis (At5g46510) (Kim et al. 2012) with two PL domains or the extreme case of the $M a$ gene in myrobalan plum, which contains five PL domains and confers a complete resistance to root-knot nematodes (Claverie et al. 2011). In such infrequent cases, the duplicated PL might play a decoy role that favors the effector detection. We can hypothesize that these duplicated PL domains could mislead effectors that target TNLs through their PL domains in a similar process described for kinases and pseudo-kinases targeted by Xanthomonas and Pseudomonas effectors in Arabidopsis (Wang et al. 2015).

\section{Loss of PL motifs in sensor TNLs.}

When overexpressed alone, many plant TIR domains from TNLs trigger HR (Bernoux et al. 2011; Schreiber et al. 2016; Williams et al. 2014; Zhang et al. 2017). It is assumed that the TIR domain functions as an executive platform for immune signaling after TNL intramolecular restructuration following effector recognition. Interestingly RRS1 and RRS1B TIR-only domains are not able to trigger HR (Saucet et al. 2016; Williams et al. 2014). They lack the $\alpha \mathrm{D} 1-2$ helix region present on TNLs known to be potent for immune signaling activation (Williams et al. 2014). Likewise, we identified in RRS1 and RRS1B a degenerated PL domain between the LRR and WRKY domains, a section of RRS1 also known as Domain 4 (Ma et al. 2018). However, RRS1 and RRS1B do not require conserved executor-like TIR or PL domains for function. In addition, mutating the RRS1 NB domain in the P-loop does not abolish its functionality when the equivalent mutation is deleterious for RPS4 (Williams et al. 2014). Sensor TNLs display unconventional architectures, such as integrated domains, domain duplication, and domain reorganization. Consequently, the specialization of their function toward sensing molecular disturbance should inevitably be accompanied with an evolution of their protein structure and might lead to degenerated sequences of their TIR and PL domains as described in our analysis. Paired TNLs must coevolve intimately to be able to activate immunity as a precise molecular duo. The TIR domain of RRS1 interacts with RPS4 TIR for activation of the complex (Williams et al. 2014). Similarly, it has been reported that RRS1 Domain 4 (which includes RRS1 PL domain) associates in planta with RPS4 PL-CTD implying the involvement of the PL domain in TNL pairing (Ma et al. 2018). How the PL domain is involved in paired TNL-activated immunity remains to be elucidated.

Our sequence and point mutation analyses reinforce a role for the executor-like PL domain in immune signaling rather than in effector recognition. Hypothetically, the TIR and PL domains function synergistically for signaling activation considering that PL domains are only found in TNLs and that no PL motifs have been detected in CNL, RNL, or other LRR-containing proteins (Van Ghelder and Esmenjaud 2016). In addition, a remarkable breakthrough has been made recently with the published high-resolution cryo-EM structure of the TNL Roq1 (Martin et al. 2020). Roq1 directly binds the bacterial effector XopQ via its PL domain to activate a tetrameric resistosome that resembles the CNL ZAR1 wheel-like pentameric resistosome (Wang et al. 2019a). Most importantly, the PL domain of Roq1 not only recognizes the effector but may also inhibit its virulence through the insertion of a particular nucleoside recognition loop (NR loop) into the active site cleft of the effector (Martin et al. 2020). Given that the Roq1 NR loop may not be a common feature of TNLs, the PL domain may also have additional functions in other TNL models, as N or RPS4/RRS1, that remain to be clarified. Notably, our results suggest that the PL domain is involved in TNL stabilization in resting state, TNL activation, and TNL-mediated immune signaling. Future analyses focused on the intra- and intermolecular interactions involving the PL domain will provide a better understanding of its role in TNLP-mediated immunity.

\section{MATERIALS AND METHODS}

\section{Detection and characterization of PL motifs.}

We retrieved TNL peptide sequences from the Araport11 dataset of The Arabidopsis Information Resource. The functional annotation of the peptides was carried out using Interproscan (Jones et al. 2014). Regions that were immediately located after the LRR domains and did not match to any known domain were extracted. These sequences were aligned, using Muscle and T-coffee, together with PL domains of reference TNLs such as N (Q40392), Ma (CAR94514), and BS4 (AAR21295), and the PL motifs defined by Van Ghelder and Esmenjaud (2016). A subset of 68 sequences were selected and submitted to MEME 5.0.5 for motif detection (Bailey et al. 2009), with maximum number of motifs set to 4 , minimum-maximum motif 
width set to 5 to 20 , and minimum sites per motif equal to 2 . Then, the full proteome Araport11 was screened for PL motifs using FIMO (e-values $<10^{-5}$ ) and BLASTP (e-values $\left.<30\right)$ with identified PL motifs as queries. The secondary structures of the PL domains were predicted using JPred4 (Drozdetskiy et al. 2015).

\section{Sequence alignment, phylogenetic analysis, and HMM profile.}

First, 67 PL sequences (the PL sequence of AT5G18370 was removed due to an insertion of multiple acidic residues, decreasing the reliability of the alignment) together with the PL domain of $\mathrm{N}$ were aligned (using Muscle and T-coffee) and were trimmed by removing amino acids localized upstream and downstream of motifs 1 and 4, respectively. The phylogenetic tree was reconstructed, using the maximum likelihood method implemented in the PhyML program (v3.1/3.0 aLRT) available online (Dereeper et al. 2010). The JTT substitution model was selected, and the reliability for internal branch was assessed using the aLRT test (SH-like). Similarly, a phylogenetic tree was produced using the maximum parsimony method (Supplementary Fig. S6). The bootstrap values consensus tree was inferred from 500 replicates. To confirm the tree topology, we selected 51 of the 67 sequences to generate a stronger alignment. This alignment was used in MEGA7 (Kumar et al. 2016) to produce a neighbor-joining phylogenetic tree (bootstrap set to 1,000 replicates), using the JTT matrix-based method (Supplementary Fig. S6). The PL domain of the N protein was used as outgroup for each tree. Using the same alignment of 51 PL sequences, we produced a HMM profile (Supplementary Data S1) and searched for this profile in the Araport11 dataset (e-value $<10^{-5}$ ) with the HMMER v3.3.

\section{Plant material and HR tests.}

$N$. tabacum 'Xanthi' and $N$. benthamiana plants used for transient assays were grown in a growth chamber ( $16 \mathrm{~h}$ of light, $8 \mathrm{~h}$ of dark). Seeds were first sowed on soil and were placed at $4^{\circ} \mathrm{C}$ in the dark for 3 days. They were then transferred in the growth chamber at $24^{\circ} \mathrm{C}$. Three weeks after transfer, plant leaves were infiltrated with a solution made of mixed Agrobacterium tumefaciens AGL1 carrying different genetic constructs at a final concentration $\mathrm{OD}_{600}=0.5$ in distilled water. The HR was assessed 2 to 5 days postinfiltration. The figures present HR phenotypes observed repetitively (at least three times) when the positive and negative controls of the transient HR assay worked accordingly in the infiltrated leaf. For ion leakage, $1-\mathrm{cm}^{2}$ leaf disk samples were taken 2 days after infiltration and were incubated for $2 \mathrm{~h}$ in distilled water on a shaker. Then two leaf disks were placed in 24-well plates in $2 \mathrm{ml}$ of distilled water. For each A. tumefaciens solution, three biological repeats were prepared. Ion leakage measurements were taken 3,4 , and 5 days postinfiltration $(24,48$, and $72 \mathrm{~h}$ after transfer in plates, respectively). The HR and ion leakage results shown have been repeated at least three times with similar results and were taken into account only when positive and negative controls presented adequate phenotypes.

\section{Gene cloning, mutation, and chimera construction.}

All the gene cloning was performed following the Golden Gate method, using the vectors and modules provided in the Golden Gate cloning kit from The Sainsbury Laboratory, Norwich, U.K. (Engler et al. 2014). Full and partial gene sequences were amplified by PCR using Phusion polymerase (Thermo Scientific). Point mutations were inserted by integrating the desired mutation in the reverse and forward primers for the amplification of two DNA modules assembled later, using the DigLig reaction. Amplicons were separated on
$0.7 \%$ to $1 \%$ agarose gel using electrophoresis, and bands of interest were isolated using a gel extraction kit (Qiagen). Final cloning was then performed by DigLig reaction into the pICH86988 vector, which contains a T-DNA for gene expression driven by a cauliflower mosaic virus $35 \mathrm{~s}$ promoter and A. tumefaciens octopine synthase terminator. The RPS4, RRS1, $R P S 4 B$, and $R R S 1 B$ genes we used in transient assays, point mutations, and chimera construction originated from Arabidopsis thaliana accession Ws-2.

\section{Immunoblot analysis.}

We tested whether each genetic construct generated with the Golden Gate method was translated to the corresponding protein in N. benthamiana. Each construct was fused to a Cterminal FLAG tag for detection by Western blot. Fourweek-old $N$. benthamiana leaves were infiltrated with a single strain of $A$. tumefaciens carrying the genetic construct to be tested. A leaf disk of $1 \mathrm{~cm}^{2}$ of the infiltrated leaf was sampled 2 days postinfiltration, was placed in a 2-ml tube, and was flash-frozen in liquid nitrogen. Each sample was then ground in the tubes and $200 \mu \mathrm{l}$ of extraction buffer (50 mM Tris-HCl, pH 7.8, 2\% sodium dodecyl sulfate [SDS], $10 \mathrm{mM}$ dithiothreitol, $10 \%$ glycerol, Halt protease inhibitor cocktail EDTA-free (Thermo Scientific), $1 \%$ polyvinylpolypyrrolidone) was added. Tubes were placed at $4{ }^{\circ} \mathrm{C}$ on a spinning wheel for $2 \mathrm{~h}$. Tubes were centrifuged for 10 $\min$ at $15,000 \times g$ at $4^{\circ} \mathrm{C}$ and supernatant was mixed in a new tube with $4 \times$ loading buffer $(250 \mathrm{mM}$ Tris- $\mathrm{HCl}, \mathrm{pH} 6.8,0.1 \%$ SDS, $40 \%$ glycerol, $0.008 \%$ bromophenol blue, $20 \%$ $\beta$-mercaptoethanol). Each ready-to-load sample was placed in a thermomixer at $95^{\circ} \mathrm{C}$ for $5 \mathrm{~min}$. Protein samples were then separated by SDS polyacrylamide gel electrophoresis. Proteins were revealed on membrane using anti-FLAG M2peroxidase antibody (Sigma-Aldrich).

\section{ACKNOWLEDGMENTS}

We thank J. D. J. Jones for providing us the T-DNA vectors for in planta expression of AvrRps4, RRS1, RPS4, RRS1B, and RPS4B. We thank B. J. Staskawicz for sending us the plasmids containing $N$ and $P 50$ gene sequences. We acknowledge M. Youles and N. Patron from The Sainsbury Laboratory in Norwich for providing us the precious Golden Gate cloning kit.

\section{AUTHOR-RECOMMENDED INTERNET RESOURCES}

The Arabidopsis Information Resource: https://www.arabidopsis.org PhyML program, v3.1/3.0 aLRT: http://www.phylogeny.fr

\section{LITERATURE CITED}

Albert, M., Jehle, A. K., Mueller, K., Eisele, C., Lipschis, M., and Felix, G. 2010. Arabidopsis thaliana pattern recognition receptors for bacterial elongation factor Tu and flagellin can be combined to form functional chimeric receptors. J. Biol. Chem. 285:19035-19042.

Bailey, T. L., Boden, M., Buske, F. A., Frith, M., Grant, C. E., Clementi, L., Ren, J., Li, W. W., and Noble, W. S. 2009. MEME SUITE: Tools for motif discovery and searching. Nucleic Acids Res. 37 (Web Server): W202-W208

Bernoux, M., Ve, T., Williams, S., Warren, C., Hatters, D., Valkov, E., Zhang, X., Ellis, J. G., Kobe, B., and Dodds, P. N. 2011. Structural and functional analysis of a plant resistance protein TIR domain reveals interfaces for self-association, signaling, and autoregulation. Cell Host Microbe 9:200-211.

Brutus, A., Sicilia, F., Macone, A., Cervone, F., and De Lorenzo, G. 2010. A domain swap approach reveals a role of the plant wall-associated kinase 1 (WAK1) as a receptor of oligogalacturonides. Proc. Natl. Acad. Sci. U.S.A. 107:9452-9457.

Burch-Smith, T. M., Schiff, M., Caplan, J. L., Tsao, J., Czymmek, K., and Dinesh-Kumar, S. P. 2007. A novel role for the TIR domain in association with pathogen-derived elicitors. PLoS Biol. 5:e0050068. 
Caplan, J. L., Mamillapalli, P., Burch-Smith, T. M., Czymmek, K., and Dinesh-Kumar, S. P. 2008. Chloroplastic protein NRIP1 mediates innate immune receptor recognition of a viral effector. Cell 132:449-462.

Castel, B., Ngou, P. M., Cevik, V., Redkar, A., Kim, D. S., Yang, Y., Ding, P., and Jones, J. D. G. 2019. Diverse NLR immune receptors activate defence via the RPW8-NLR NRG1. New Phytol. 222:966-980.

Césari, S., Kanzaki, H., Fujiwara, T., Bernoux, M., Chalvon, V., Kawano, Y., Shimamoto, K., Dodds, P., Terauchi, R., and Kroj, T. 2014. The NBLRR proteins RGA4 and RGA5 interact functionally and physically to confer disease resistance. EMBO J. 33:1941-1959.

Césari, S., Thilliez, G., Ribot, C., Chalvon, V., Michel, C., Jauneau, A., Rivas, S., Alaux, L., Kanzaki, H., Okuyama, Y., Morel, J.-B., Fournier, E., Tharreau, D., Terauchi, R., and Kroj, T. 2013. The rice resistance protein pair RGA4/RGA5 recognizes the Magnaporthe oryzae effectors AVR-Pia and AVR1-CO39 by direct binding. Plant Cell 25:1463-1481.

Claverie, M., Dirlewanger, E., Bosselut, N., Van Ghelder, C., Voisin, R., Kleinhentz, M., Lafargue, B., Abad, P., Rosso, M. N., Chalhoub, B., and Esmenjaud, D. 2011. The $M a$ gene for complete-spectrum resistance to Meloidogyne species in Prunus is a TNL with a huge repeated Cterminal post-LRR region. Plant Physiol. 156:779-792.

Cui, H., Tsuda, K., and Parker, J. E. 2015. Effector-triggered immunity: From pathogen perception to robust defense. Annu. Rev. Plant Biol. 66: 487-511.

Dereeper, A., Audic, S., Claverie, J. M., and Blanc, G. 2010. BLASTEXPLORER helps you building datasets for phylogenetic analysis. BMC Evol. Biol. 10:8-13.

Deslandes, L., Olivier, J., Peeters, N., Feng, D. X., Khounlotham, M., Boucher, C., Somssich, I., Genin, S., and Marco, Y. 2003. Physical interaction between RRS1-R, a protein conferring resistance to bacterial wilt, and PopP2, a type III effector targeted to the plant nucleus. Proc. Natl. Acad. Sci. U.S.A. 100:8024-8029.

Dodds, P. N., Lawrence, G. J., and Ellis, J. G. 2001. Six amino acid changes confined to the leucine-rich repeat $\beta$-strand/ $\beta$-turn motif determine the difference between the $\mathrm{P}$ and $\mathrm{P} 2$ rust resistance specificities in flax. Plant Cell 13:163-178.

Dong, O. X., Tong, M., Bonardi, V., El Kasmi, F., Woloshen, V., Wünsch, L. K., Dangl, J. L., and Li, X. 2016. TNL-mediated immunity in Arabidopsis requires complex regulation of the redundant ADRI gene family. New Phytol. 210:960-973.

Drozdetskiy, A., Cole, C., Procter, J., and Barton, G. J. 2015. JPred4: A protein secondary structure prediction server. Nucleic Acids Res. 43 (W1):W389-W394.

Engler, C., Youles, M., Gruetzner, R., Ehnert, T. M., Werner, S., Jones, J. D. G., Patron, N. J., and Marillonnet, S. 2014. A golden gate modular cloning toolbox for plants. ACS Synth. Biol. 3:839-843.

Gao, Y., Wang, W., Zhang, T., Gong, Z., Zhao, H., and Han, G.-Z. 2018. Out of water: The origin and early diversification of plant $R$-genes. Plant Physiol. 177:82-89.

Gassmann, W., Hinsch, M. E., and Staskawicz, B. J. 1999. The Arabidopsis RPS4 bacterial-resistance gene is a member of the TIR-NBS-LRR family of disease-resistance genes. Plant J. 20:265-277.

Heath, M. C. 2000. Hypersensitive response-related death. Plant Mol. Biol. 44:321-334

Holton, N., Nekrasov, V., Ronald, P. C., and Zipfel, C. 2015. The phylogenetically-related pattern recognition receptors EFR and XA21 recruit similar immune signaling components in monocots and dicots. PLoS Pathog. 11:e1004602.

Howles, P., Lawrence, G., Finnegan, J., McFadden, H., Ayliffe, M., Dodds, P., and Ellis, J. 2005. Autoactive alleles of the flax L6 rust resistance gene induce non-race-specific rust resistance associated with the hypersensitive response. Mol. Plant-Microbe Interact. 18:570-582.

Huh, S. U., Cevik, V., Ding, P., Duxbury, Z., Ma, Y., Tomlinson, L., Sarris, P. F., and Jones, J. D. G. 2017. Protein-protein interactions in the RPS4/RRS1 immune receptor complex. PLoS Pathog. 13:e1006376.

Jones, J. D. G., and Dangl, J. L. 2006. The plant immune system. Nature 444:323-329.

Jones, P., Binns, D., Chang, H. Y., Fraser, M., Li, W., McAnulla, C., McWilliam, H., Maslen, J., Mitchell, A., Nuka, G., Pesseat, S., Quinn, A. F., Sangrador-Vegas, A., Scheremetjew, M., Yong, S. Y., Lopez, R., and Hunter, S. 2014. InterProScan 5: Genome-scale protein function classification. Bioinformatics 30:1236-1240.

Kim, T.-H., Kunz, H.-H., Bhattacharjee, S., Hauser, F., Park, J., Engineer, C., Liu, A., Ha, T., Parker, J. E., Gassmann, W., and Schroeder, J. I. 2012. Natural variation in small molecule-induced TIR-NB-LRR signaling induces root growth arrest via EDS1- and PAD4-complexed R protein VICTR in Arabidopsis. Plant Cell 24:5177-5192.

Kroj, T., Chanclud, E., Michel-Romiti, C., Grand, X., and Morel, J. B. 2016. Integration of decoy domains derived from protein targets of pathogen effectors into plant immune receptors is widespread. New Phytol. 210: 618-626.

Kumar, S., Stecher, G., and Tamura, K. 2016. MEGA7: Molecular evolutionary genetics analysis version 7.0 for bigger datasets. Mol Biol. Evol. 33:1870-1874.

Le Roux, C., Huet, G., Jauneau, A., Camborde, L., Trémousaygue, D., Kraut, A., Zhou, B., Levaillant, M., Adachi, H., Yoshioka, H., Raffaele, S., Berthomé, R., Couté, Y., Parker, J. E., and Deslandes, L. 2015. A receptor pair with an integrated decoy converts pathogen disabling of transcription factors to immunity. Cell 161:1074-1088.

Ma, Y., Guo, H., Hu, L., Martinez, P. P., Moschou, P. N., Cevik, V., Ding, P., Duxbury, Z., Sarris, P. F., and Jones, J. D. G. 2018. Distinct modes of derepression of an Arabidopsis immune receptor complex by two different bacterial effectors. Proc. Natl. Acad. Sci. U.S.A. 115 10218-10227.

Martin, R., Qi, T., Zhang, H., Liu, F., King, M., Toth, C., Nogales, E., and Staskawicz, B. J. 2020. Structure of the activated Roq1 resistosome directly recognizing the pathogen effector XopQ. Science 370 eabd9993.

Meyers, B. C., Kozik, A., Griego, A., Kuang, H., and Michelmore, R. W 2003. Genome-wide analysis of NBS-LRR-encoding genes in Arabidopsis. Plant Cell 15:809-834.

Meyers, B. C., Morgante, M., and Michelmore, R. W. 2002. TIR-X and TIR-NBS proteins: Two new families related to disease resistance TIRNBS-LRR proteins encoded in Arabidopsis and other plant genomes. Plant J. 32:77-92.

Narusaka, M., Kubo, Y., Hatakeyama, K., Imamura, J., Ezura, H., Nanasato, Y., Tabei, Y., Takano, Y., Shirasu, K., and Narusaka, Y. 2013. Breaking restricted taxonomic functionality by dual resistance genes. Plant Signal. Behav. 8:e24244.

Narusaka, M., Shirasu, K., Noutoshi, Y., Kubo, Y., Shiraishi, T., Iwabuchi, M., and Narusaka, Y. 2009. RRS1 and RPS4 provide a dual Resistancegene system against fungal and bacterial pathogens. Plant J. 60:218-226.

Peart, J. R., Mestre, P., Lu, R., Malcuit, I., and Baulcombe, D. C. 2005. NRG1, a CC-NB-LRR protein, together with N, a TIR-NB-LRR protein, mediates resistance against tobacco mosaic virus. Curr. Biol. 15: 968-973.

Qi, T., Seong, K., Thomazella, D. P. T., Kim, J. R., Pham, J., Seo, E., Cho, M. J., Schultink, A., and Staskawicz, B. J. 2018. NRG1 functions downstream of EDS1 to regulate TIR-NLR-mediated plant immunity in Nicotiana benthamiana. Proc. Natl. Acad. Sci. U.S.A. 115:E10979-E10987.

Rairdan, G. J., and Moffett, P. 2006. Distinct domains in the ARC region of the potato resistance protein Rx mediate LRR binding and inhibition of activation. Plant Cell 18:2082-2093.

Sarris, P. F., Duxbury, Z., Huh, S. U., Ma, Y., Segonzac, C., Sklenar, J., Derbyshire, P., Cevik, V., Rallapalli, G., Saucet, S. B., Wirthmueller, L., Menke, F. L. H., Sohn, K. H., and Jones, J. D. G. 2015. A plant immune receptor detects pathogen effectors that target WRKY transcription factors. Cell 161:1089-1100.

Saucet, S. B., Ma, Y., Sarris, P. F., Furzer, O. J., Sohn, K. H., and Jones, J. D. G. 2015. Two linked pairs of Arabidopsis TNL resistance genes independently confer recognition of bacterial effector AvrRps4. Nat. Commun. 6:6338.

Schreiber, K. J., Bentham, A., Williams, S. J., Kobe, B., and Staskawicz, B. J. 2016. Multiple domain associations within the Arabidopsis immune receptor RPP1 regulate the activation of programmed cell death. PLoS Pathog. 12:e1005769.

Shao, Z.-Q., Xue, J.-Y., Wu, P., Zhang, Y.-M., Wu, Y., Hang, Y.-Y., Wang, B., and Chen, J.-Q. 2016. Large-scale analyses of angiosperm nucleotidebinding site-leucine-rich repeat genes reveal three anciently diverged classes with distinct evolutionary patterns. Plant Physiol. 170:2095-2109.

Sohn, K. H., Segonzac, C., Rallapalli, G., Sarris, P. F., Woo, J. Y., Williams, S. J., Newman, T. E., Paek, K. H., Kobe, B., and Jones, J. D. G. 2014. The nuclear immune receptor RPS4 is required for RRS1SLH1-dependent constitutive defense activation in Arabidopsis thaliana. PLoS Genet. 10: e1004655.

Swiderski, M. R., Birker, D., and Jones, J. D. G. 2009. The TIR domain of TIR-NB-LRR resistance proteins is a signaling domain involved in cell death induction. Mol. Plant-Microbe Interact. 22:157-165.

Takken, F. L., Albrecht, M., and Tameling, W. I. L. 2006. Resistance proteins: Molecular switches of plant defence. Curr. Opin. Plant Biol. 9: 383-390.

Takken, F. L. W., and Goverse, A. 2012. How to build a pathogen detector: Structural basis of NB-LRR function. Curr. Opin. Plant Biol. 15:375-384.

Tarr, D. E. K., and Alexander, H. M. 2009. TIR-NBS-LRR genes are rare in monocots: Evidence from diverse monocot orders. BMC Res. Notes 2:197.

Ueda, H., Yamaguchi, Y., and Sano, H. 2006. Direct interaction between the tobacco mosaic virus helicase domain and the ATP-bound resistance 
protein, $\mathrm{N}$ factor during the hypersensitive response in tobacco plants. Plant Mol. Biol. 61:31-45.

Van Ghelder, C., and Esmenjaud, D. 2016. TNL genes in peach: Insights into the post-LRR domain. BMC Genomics 17:317.

Van Ghelder, C., Parent, G. J., Rigault, P., Prunier, J., Giguère, I., Caron, S., Stival Sena, J., Deslauriers, A., Bousquet, J., Esmenjaud, D., and MacKay, J. 2019. The large repertoire of conifer NLR resistance genes includes drought responsive and highly diversified RNLs. Sci. Rep. 9:11614.

Wan, L., Essuman, K., Anderson, R. G., Sasaki, Y., Monteiro, F., Chung, E. H., Nishimura, E. O., DiAntonio, A., Milbrandt, J., Dangl, J. L., and Nishimura, M. T. 2019. TIR domains of plant immune receptors are $\mathrm{NAD}^{+}$-cleaving enzymes that promote cell death. Science 365:799-803.

Wang, G., Roux, B., Feng, F., Guy, E., Li, L., Li, N., Zhang, X., Lautier, M., Jardinaud, M. F., Chabannes, M., Arlat, M., Chen, S., He, C., Noël, L. D., and Zhou, J. M. 2015. The decoy substrate of a pathogen effector and a pseudokinase specify pathogen-induced modified-self recognition and immunity in plants. Cell Host Microbe 18:285-295.

Wang, J., Hu, M., Wang, J., Qi, J., Han, Z., Wang, G., Qi, Y., Wang, H. W., Zhou, J. M., and Chai, J. 2019a. Reconstitution and structure of a plant NLR resistosome conferring immunity. Science 364:aav5870.

Wang, J., Wang, J., Hu, M., Wu, S., Qi, J., Wang, G., Han, Z., Qi, Y., Gao, N., Wang, H. W., Zhou, J. M., and Chai, J. 2019b. Ligand-triggered allosteric ADP release primes a plant NLR complex. Science 364:aav5868

Whitham, S., Dinesh-Kumar, S. P., Choi, D., Hehl, R., Corr, C., and Baker, B. 1994. The product of the tobacco mosaic virus resistance gene $N$ : Similarity to toll and the interleukin-1 receptor. Cell 78:1101-1115.
Williams, S. J., Sohn, K. H., Wan, L., Bernoux, M., Sarris, P. F., Segonzac, C., Ve, T., Ma, Y., Saucet, S. B., Ericsson, D. J., Casey, L. W., Lonhienne, T., Winzor, D. J., Zhang, X., Coerdt, A., Parker, J. E., Dodds, P. N., Kobe, B., and Jones, J. D. G. 2014. Structural basis for assembly and function of a heterodimeric plant immune receptor. Science 344:299-303.

Wirthmueller, L., Zhang, Y., Jones, J. D. G., and Parker, J. E. 2007. Nuclear accumulation of the Arabidopsis immune receptor RPS4 is necessary for triggering EDS1-dependent defense. Curr. Biol. 17:2023-2029.

Wu, C. H., Abd-El-Haliem, A., Bozkurt, T. O., Belhaj, K., Terauchi, R., Vossen, J. H., and Kamoun, S. 2017. NLR network mediates immunity to diverse plant pathogens. Proc. Natl. Acad. Sci. U.S.A. 114:8113-8118.

Xue, J.-Y., Wang, Y., Wu, P., Wang, Q., Yang, L.-T., Pan, X.-H., Wang, B., and Chen, J.-Q. 2012. A primary survey on bryophyte species reveals two novel classes of nucleotide-binding site (NBS) genes. PLoS One 7: e36700.

Yue, J. X., Meyers, B. C., Chen, J. Q., Tian, D., and Yang, S. 2012. Tracing the origin and evolutionary history of plant nucleotide-binding siteleucine-rich repeat (NBS-LRR) genes. New Phytol. 193:1049-1063.

Zhang, X., Bernoux, M., Bentham, A. R., Newman, T. E., Ve, T., Casey, L. W., Raaymakers, T. M., Hu, J., Croll, T. I., Schreiber, K. J., Staskawicz, B. J., Anderson, P. A., Sohn, K. H., Williams, S. J., Dodds, P. N., and Kobe, B. 2017. Multiple functional self-association interfaces in plant TIR domains. Proc. Natl. Acad. Sci. U.S.A. 114:E2046-E2052.

Zhang, Y., Dorey, S., Swiderski, M., and Jones, J. D. G. 2004. Expression of RPS4 in tobacco induces an AvrRps4-independent HR that requires EDS1, SGT1 and HSP90. Plant J. 40:213-224. 